\title{
On an analytic approach to the Fatou conjecture
}

\author{
by
}

Genadi Levin (Jerusalem)

\begin{abstract}
Let $f$ be a quadratic map (more generally, $f(z)=z^{d}+c, d>1$ ) of the complex plane. We give sufficient conditions for $f$ to have no measurable invariant linefields on its Julia set. We also prove that if the series $\sum_{n \geq 0} 1 /\left(f^{n}\right)^{\prime}(c)$ converges absolutely, then its sum is non-zero. In the proof we use analytic tools, such as integral and transfer (Ruelle-type) operators and approximation theorems.
\end{abstract}

1. Statements and remarks. The Fatou conjecture says that every rational map can be approximated by hyperbolic rational maps. We focus on the family of quadratic polynomials. It follows from a general result [MSS] that a quadratic polynomial $f$ can be approximated by hyperbolic quadratic polynomials if and only if $f$ has no (measurable) invariant linefield on its Julia set. Thus the Fatou conjecture is reduced to the problem whether $f$ can carry such a linefield. For the quadratic family, the Fatou conjecture is also equivalent to the following conjectural picture: every component of the interior of the Mandelbrot set consists of hyperbolic maps; the latter would also follow from a stronger MLC ("Mandelbrot set is locally connected") conjecture $[\mathrm{DH}]$.

In what follows we fix a quadratic polynomial $f(z)=z^{2}+c$ such that the Julia set $J$ of $f$ is connected, and $f$ has no attracting or parabolic periodic orbits. (For the basic definitions and necessary background see e.g. [CG], [Mc].)

Denote by

$$
c_{0}=0, \quad c_{1}=f(0)=c, \quad c_{2}=f^{2}(0), \ldots, c_{n}=f^{n}(0), \ldots
$$

the forward iterates of the critical point $c_{0}=0$. By the assumptions, all $c_{n}$ are in $J$.

2000 Mathematics Subject Classification: 37F10, 30D05, 37C30.

Preprint versions of this article were circulating since December, 2000. Corollary 1(b) and Theorem 2 were included in the January 2001 and May 2001 versions respectively. 
Introduce the following sequence of (complex) measures $\nu_{n}$. Let $\delta_{x}$ be the measure of mass 1 concentrated at the point $x$ (Dirac's measure). For any $n>0$, set

$$
\nu_{n}=\frac{1}{\Delta_{n}} \sum_{k=1}^{n} \frac{1}{\left(f^{k-1}\right)^{\prime}(c)} \delta_{c_{k}},
$$

where

$$
\Delta_{n}=1+\frac{1}{\left|f^{\prime}(c)\right|}+\ldots+\frac{1}{\left|\left(f^{n-1}\right)^{\prime}(c)\right|},
$$

so that the total variation of the measure $\nu_{n}$ is 1 .

TheOrem 1. Assume that $f$ admits an invariant linefield on its Julia set $J$. Assume that

$$
\left(f^{n_{i}-1}\right)^{\prime}(c) \Delta_{n_{i}} \rightarrow \infty
$$

along a sequence $n_{i} \rightarrow \infty$. Then the sequence of measures $\nu_{n_{i}}$ tends to zero in the weak topology.

If $c_{n_{i}} \rightarrow c_{0}$ then $\nu_{n_{i}} \rightarrow 0$ without the assumption (*) (which holds automatically in this case).

Note that

$$
\left|\left(f^{n_{i}-1}\right)^{\prime}(c)\right| \Delta_{n_{i}}=1+\sum_{k=1}^{n_{i}-1}\left|\left(f^{k}\right)^{\prime}\left(c_{n_{i}-k}\right)\right| .
$$

We do not know of any example of a non-hyperbolic quadratic map for which $(*)$ is not true. (Natural candidates are maps with non-locally connected Julia sets.) Note however that condition $(*)$ arises naturally in the proof of Theorem 1, so it makes sense to study it separately. It turns out that the same method as in the proof of Theorem 1 works (in the case $c_{n_{i}} \rightarrow c_{0}$ ) to prove that if $(*)$ does not hold then $f$ has no linefields (see Remark 3 and Theorem 2).

REMARK 1. Each $\nu_{n}$ is a discrete complex measure of total variation 1. Therefore, $\nu_{n_{i}} \rightarrow 0$ means (informally speaking) that there are a lot of cancellations in the limit.

If the increasing sequence $\Delta_{n}, n>0$, is bounded (i.e. the series $\sum_{n \geq 0} 1 /\left|\left(f^{n}\right)^{\prime}(c)\right|$ converges to a finite limit $\left.\Delta\right)$ then $\left(f^{n}\right)^{\prime}(c) \rightarrow \infty$, and, by Theorem 1, if $f$ has an invariant linefield, then the sequence $\nu_{n}$ converges to the zero measure. On the other hand, in this case $\nu_{n}$ converges to the measure

$$
\nu=\frac{1}{\Delta} \sum_{k=1}^{\infty} \frac{1}{\left(f^{k-1}\right)^{\prime}(c)} \delta_{c_{k}},
$$

which is non-zero (it is purely atomic). Therefore, we have the first part of the following 
Corollary 1. Assume that the series

$$
\sum_{n \geq 1} \frac{1}{\left|\left(f^{n-1}\right)^{\prime}(c)\right|}
$$

converges. Then:

(a) $f$ has no invariant linefields on its Julia set.

(b) the series

$$
\sum_{n \geq 1} \frac{1}{\left(f^{n-1}\right)^{\prime}(c)}
$$

converges to a non-zero number $S_{*} \neq 0$.

REMARK 2. Corollary 1(a) is not new: in [BS] and in [R-L] stronger results are shown, which imply in particular that the area of $J$ is zero. Moreover, in $[\mathrm{R}-\mathrm{L}]$ it is proved that the Mandelbrot set is locally connected at such $c$. Note however that the methods of [BS], [R-L] are very different from ours: the authors use sharp distortion estimates as well as probabilistic and geometric considerations.

Let us comment on part (b) of Corollary 1. Although our proof of Corollary 1 (b) is analytic, it has the following geometrical interpretation. Denote by $c_{n}^{\prime}$ the derivative of $f^{n}(0)$ (which is a polynomial in $c$ ) with respect to the parameter $c$ at the point $c$. Then the statement of Corollary 1(b) means that $c_{n}^{\prime} /\left(f^{n-1}\right)^{\prime}(c) \rightarrow S_{*} \neq 0$ as $n \rightarrow \infty$.

A particular case of Corollary 1(b) (namely, for $c$ such that the series $\sum_{n>0}\left|\left(f^{n}\right)^{\prime}(c)\right|^{-1 / 2}$ converges) is proved in [R-L] (by geometrical considerations). The quantity $S_{*}$ arises naturally in [R-L] as a similarity factor between the Mandelbrot set around such a $c$, and the corresponding Julia set.

See also Section 4, Remark 13.

REMARK 3. Having Corollary 1 , we can assume in Theorem 1 that $\Delta_{n} \rightarrow$ $\infty$. In particular, condition $(*)$ holds if the sequence $\left(f^{n_{i}-1}\right)^{\prime}(c)$ stays away from zero. Moreover, the following statement shows that if $c_{n_{i}} \rightarrow c_{0}$ and condition $(*)$ does not hold then $f$ has no invariant linefields. (This implies, for example, that if $c_{n_{i}} \rightarrow c_{0}$ then $(*)$ can be dropped in the statement of Theorem 1). Note in this regard that if $c_{n_{i}}$ does not tend to the critical point $c_{0}$ for any sequence $n_{i}$ (i.e. $c_{0}$ is not recurrent) then it is well known that $f$ has no invariant linefields.

Theorem 2. (a) If $c_{n_{i}} \rightarrow c_{0}$ and the sequence $\left(f^{n_{i}-1}\right)^{\prime}(c) \Delta_{n_{i}}$ does not tend to infinity, then $f$ has no invariant linefields.

(b) Let $\left(c_{-n}\right)_{n \geq 1}$ be a sequence of pre-images of the critical point $c_{0}=0$ :

$$
f\left(c_{-n}\right)=c_{-(n-1)}, \quad n=1,2, \ldots
$$


If

$$
\sum_{n=1}^{\infty}\left|\left(f^{n}\right)^{\prime}\left(c_{-n}\right)\right|<\infty
$$

then $f$ has no invariant linefields, and moreover,

$$
S^{*}:=1+\sum_{n=1}^{\infty}\left(f^{n}\right)^{\prime}\left(c_{-n}\right) \neq 0 .
$$

REMARK 4. $S^{*} \neq 0$ has similar geometric meaning to $S_{*} \neq 0$. Namely, $c_{-n}^{\prime}\left(f^{n}\right)^{\prime}\left(c_{-n}\right) \rightarrow-S^{*} \neq 0$ as $n \rightarrow \infty$, where $c_{-n}^{\prime}$ is the derivative with respect to $c$.

We prove that similar results hold for renormalizations of $f$. Given a set $E$, define

$$
\nu_{n, E}=\frac{1}{\Delta_{n, E}} \sum_{1 \leq k \leq n, c_{k} \in E} \frac{1}{\left(f^{k-1}\right)^{\prime}(c)} \delta_{c_{k}},
$$

where

$$
\Delta_{n, E}=\sum_{1 \leq k \leq n, c_{k} \in E} \frac{1}{\left|\left(f^{k-1}\right)^{\prime}(c)\right|} .
$$

Then we have:

THEOREM 1'. Assume that $f$ admits an invariant linefield on its Julia set $J$. Let $J^{\prime} \subset J$ be the Julia set of a renormalization $f^{p}: J^{\prime} \rightarrow J^{\prime}$ of $f$. If $\left(f^{n_{i}-1}\right)^{\prime}(c) \Delta_{n_{i}, J^{\prime}} \rightarrow \infty$ for a sequence $n_{i} \rightarrow \infty$, then the sequence of measures $\nu_{n_{i}, J^{\prime}}$ tends to zero in the weak topology.

As above, we have

Corollary 1'. Assume that the series

$$
\sum_{n \geq 1, c_{n} \in J^{\prime}} \frac{1}{\left|\left(f^{n-1}\right)^{\prime}(c)\right|}
$$

converges. Then $f$ has no invariant linefields on its Julia set.

We derive Theorems 1 and $1^{\prime}$ from the propositions below.

Introduce the following notations:

$$
S_{1}=1, \quad S_{n}=1+\frac{1}{f^{\prime}(c)}+\ldots+\frac{1}{\left(f^{n-1}\right)^{\prime}(c)}, \quad n>1 ;
$$

let $Q\left(c_{0}\right)=0, Q\left(c_{1}\right)=1$, and in general

$$
Q\left(c_{n}\right)=\left(f^{n-1}\right)^{\prime}(c) S_{n}=\left(f^{n-1}\right)^{\prime}(c)\left(1+\frac{1}{f^{\prime}(c)}+\ldots+\frac{1}{\left(f^{n-1}\right)^{\prime}(c)}\right) .
$$

For the meaning of the values $Q\left(c_{n}\right)$, see Remark 6(a).

The following statements are of independent interest. 
Proposition 1. Assume that $f$ admits a non-trivial invariant linefield on its Julia set J. Then:

(a) there exists $M_{1}$ such that

$$
\left|Q\left(c_{n}\right)\right| \leq M_{1}, \quad n=1,2, \ldots ;
$$

(b) the sequence $Q\left(c_{n}\right)$ extends to a continuous function $Q$ on the plane which has the following properties:

- there is a constant $K$ (which depends only on $f$ ) so that, for every $x, y \in \mathbb{C}$,

$$
|Q(x)-Q(y)| \leq K|x-y| \log |x-y|^{-1}
$$

- $Q$ is holomorphic outside $J$;

- for every point $z$ of the plane such that $\left(f^{n}\right)^{\prime}(z) \rightarrow \infty$ as $n \rightarrow \infty$,

$$
Q(z)=-\sum_{n=1}^{\infty} \frac{1}{\left(f^{n}\right)^{\prime}(z) .}
$$

Proposition 1 immediately implies

Proposition 2. Assume that $f$ has an invariant linefield on J. Then the function which is defined outside $J$ by the latter formula extends uniquely to a continuous function on the plane, namely, to the function $Q$.

Note that the geometrical meaning of Propositions 1-2 will become transparent in view of Remark 12 of Section 2.

COROllary 2. Assume that $f$ has a non-trivial invariant linefield on $J$. If $\left(f^{n_{i}}\right)^{\prime}(c) \rightarrow \infty$ along a sequence $n_{i} \rightarrow \infty$, then

$$
\left|S_{n_{i}+1}\right|=\left|1+\frac{1}{f^{\prime}(c)}+\ldots+\frac{1}{\left(f^{n_{i}}\right)^{\prime}(c)}\right| \leq \frac{M_{1}}{\left|\left(f^{n_{i}}\right)^{\prime}(c)\right|} \rightarrow 0 .
$$

In particular, if $\left(f^{n}\right)^{\prime}(c) \rightarrow \infty$ as $n \rightarrow \infty$, then

$$
1+\frac{1}{f^{\prime}(c)}+\ldots+\frac{1}{\left(f^{n}\right)^{\prime}(c)}+\ldots=0 .
$$

Remarks. 5. The approach in Section 2 is somewhat similar to [Mak], where probably for the first time the function $\widehat{\mu}$ is used to study the problem. A difference with [Mak] is that I derive and use a functional equation for $\widehat{\mu}$ (see Section 2; Proposition 1 of this paper was proved before I became acquainted with $[\mathrm{Mak}])$.

Proposition 1 strengthens Theorem B of [Mak] (being applied to the quadratic polynomials).

6. Note that part (a) of Proposition 1 (but not part (b) or Proposition 2 or Proposition $3^{\prime}(\mathrm{b})$ below) follows immediately from the following two facts, where the first one is easy to verify, and the second one is well known: 
(a) $Q\left(c_{n}\right)=c_{n}^{\prime}$, where, as in Remark $2, c_{n}^{\prime}$ denotes the derivative of $f^{n}(0)$ with respect to $c$;

(b) the family of polynomials $f^{n}(0)$ is normal at $c$ (if $f$ has an invariant linefield).

7. All the results (except for Propositions 1 and 2) and their proofs hold without any changes if we replace the assumption: " $f$ has an invariant linefield on its Julia set" by a weaker assumption: "the sequence $Q\left(c_{n}\right)$ is bounded" (for the meaning of the values $Q\left(c_{n}\right)$, see Remark 6(a)).

8. Our approach carries over to non-quadratic maps as well. More exactly, all results and proofs hold for $z^{d}+c, d>1$. (Then, in Theorem $1^{\prime}$, the set $J^{\prime}$ can be the Julia set of a generalized renormalization as well.) For other rational maps, some modifications of the method are needed.

To prove the main results of the paper, i.e. Theorems 1 and $1^{\prime}$, and Corollary 1(b), we introduce and study the following sequence of functions: for every $n \geq 1$, let

$$
\phi_{n}(z)=\frac{1}{z-c_{1}}+\frac{1}{f^{\prime}(c)\left(z-c_{2}\right)}+\ldots+\frac{1}{\left(f^{n-1}\right)^{\prime}(c)\left(z-c_{n}\right)},
$$

which is a holomorphic function outside $J$.

Proposition 3. Assume that $f$ has an invariant linefield on $J$.

(a) Let $V$ be a neighborhood of $J$ such that $U=f^{-1}(V)$ is contained in $V$. There exists a constant $M_{2}$, which depends only on $f$ and $V$, such that, for all $n$,

$$
\int_{V \backslash U}\left|\phi_{n}(z)\right| d \sigma_{z} \leq \frac{M_{2}}{\left|\left(f^{n-1}\right)^{\prime}(c)\right|} .
$$

Here $d \sigma$ is the Lebesgue measure on the plane.

(b) For every compact set $K$ on the plane such that $K \cap J=\emptyset$, there exists a constant $M_{3}$, depending only on $f$ and $K$, such that, for all $n$ and every $z \in K$,

$$
\left|\phi_{n}(z)\right| \leq \frac{M_{3}}{\left|\left(f^{n-1}\right)^{\prime}(c)\right|} .
$$

In particular,

Corollary 3. Assume that $f$ has an invariant linefield on its Julia set $J$. Assume also that $\left(f^{n_{i}-1}\right)^{\prime}(c) \rightarrow \infty$ along a sequence $n_{i} \rightarrow \infty$. Then the sequence of functions $\phi_{n_{i}}$ tends to zero uniformly on compact subsets of $\mathbb{C} \backslash J$.

Making use of the same ideas as in the proof of Proposition 3, we can substantially refine the statements above as follows. Roughly speaking, we show that all statements hold for the renormalizations of $f$. To be more 
precise, let $\omega$ denote the $\omega$-limit set of the critical point of $f$. Call a bounded domain $\Omega$ on the plane a $Y$-domain if the boundary of $\Omega$ intersects the Julia set $J$ at finitely many points $a$, and every such $a$ is (pre)periodic under $f$. (One can assume that $\partial \Omega$ outside $J$ consists of finitely many arcs of external rays and equipotentials of $f$; in what follows, Y-domains will be finite unions of Yoccoz puzzle pieces of $f$.)

REMARK 9. It follows from a theorem of Yoccoz (see $[\mathrm{H}]$ ) that a quadratic polynomial with an invariant linefield has to be infinitely renormalizable. For an appropriate construction of the puzzle structure for infinitely renormalizable maps see e.g. [Le]. On the other hand, our approach carries over to non-quadratic maps as well (see Remark 8).

Proposition 4. Assume that $f$ has an invariant linefield on J. Then for every $Y$-domain $\Omega$ with closure disjoint from $\omega$, there exists a constant $M_{5}$, depending only on $f$ and $\Omega$, such that, for all $n$ and every $z \in \Omega$,

$$
\left|\phi_{n}(z)\right| \leq \frac{M_{5}}{\left|\left(f^{n-1}\right)^{\prime}(c)\right|} .
$$

To put the latter statement in the right context, observe that the compact set $\omega \subset J$ is disjoint from the set of all (pre)periodic points of $f$ (the latter set is dense in $J$ ). Indeed, it is known that otherwise $J$ has zero area. In particular, the boundary of the Y-domain $\Omega$ is always disjoint from $\omega$, and we can assume in Proposition 4 that $\Omega$ itself is disjoint from $\omega$. It also shows that $\omega$ is a proper subset of $J$. More exactly, denote by $Y(\omega)$ the intersection of all covers of $\omega$ by puzzle pieces. Then the compact set $Y(\omega) \subset J$ is also disjoint from the set of (pre)periodic points of $f$. Note that if $J$ happens to be locally connected, then $Y(\omega)=\omega$ and $\omega$ is a Cantor set (otherwise the boundary of a puzzle piece would intersect an arc in $\omega$ at a (pre)periodic point).

Let us draw two corollaries.

Corollary 4. Assume that $f$ has an invariant linefield on J. For every compact set $K$ on the plane such that $K \cap Y(\omega)=\emptyset$, there exists a constant $M$, depending only on $f$ and $K$, such that, for all $n$ and every $z \in K$,

$$
\left|\phi_{n}(z)\right| \leq \frac{M}{\left|\left(f^{n-1}\right)^{\prime}(c)\right|} .
$$

In turn, this implies that the same estimates hold for parts of $\phi_{n}$ corresponding to renormalizations of $f$ !

Namely, given a set $E$, define

$$
\begin{aligned}
\phi_{n, E}(z) & =\sum_{1 \leq k \leq n, c_{k} \in E} \frac{1}{\left(f^{k-1}\right)^{\prime}(c)\left(z-c_{k}\right)}, \\
S_{n, E} & =\sum_{1 \leq k \leq n, c_{k} \in E} \frac{1}{\left(f^{k-1}\right)^{\prime}(c)} .
\end{aligned}
$$


Proposition $3^{\prime}$. Assume that $f$ has an invariant linefield on $J$. Let $J^{\prime} \subset J$ be the Julia set of a renormalization $f^{p}: J^{\prime} \rightarrow J^{\prime}$ of $f$. Then:

(a) For every compact set $K$ on the plane such that $K \cap J^{\prime}=\emptyset$, there exists a constant $M_{6}$ such that, for all $n$ and every $z \in K$,

$$
\left|\phi_{n, J^{\prime}}(z)\right| \leq \frac{M_{6}}{\left|\left(f^{n-1}\right)^{\prime}(c)\right|} .
$$

(b) There exists a constant $M_{7}$ such that, for every $n$,

$$
\left|S_{n, J^{\prime}}\right| \leq \frac{M_{7}}{\left|\left(f^{n-1}\right)^{\prime}(c)\right|}
$$

Remark 10. For example, if $\left(f^{n}\right)^{\prime}(c) \rightarrow \infty$, then not only the sum $\sum_{n \geq 0} 1 /\left(f^{n}\right)^{\prime}(c)$ is zero, but any subseries with indices corresponding to a renormalization of $f$ also has zero sum. Note that it is not clear how to derive all these properties for the renormalization of the map $f$ using the corresponding properties of another quadratic map to which the renormalization of $f$ is quasi-conformally conjugate.

Acknowledgements. I thank Peter Makienko for useful discussions and for pointing out Lemma 7; Alex Eremenko, Benjamin Weiss, Feliks Przytycki for useful discussions; and Sebastian van Strien for careful reading of part of the manuscript and for stimulating and helpful comments. I would also like to thank the referee for suggesting a shorter proof of Lemma 6 . Part of this work was done during my visit to the Institute of Mathematics, Polish Academy of Sciences, Warsaw (first half of October, 2000). I thank the Institute for invitation and hospitality.

2. Proof of Proposition 1. Assume that $f$ does have a measurable invariant linefield on $J$. Then the area of $J$ is positive, and there is a function $\mu$ defined almost everywhere on $J$ such that

$$
\begin{gathered}
|\mu(z)|=1 \quad \text { on a subset of } J \text { of positive measure, } \\
\mu(f(z))=\left(f^{\prime}(z) /\left|f^{\prime}(z)\right|\right)^{2} \mu(z) \quad \text { a.e. }
\end{gathered}
$$

Define also

$$
\mu=0 \quad \text { off } J .
$$

Consider the so-called Cauchy transform of $\mu$ :

$$
\widehat{\mu}(z)=\int \frac{\mu(w)}{z-w} d \sigma_{w},
$$

where the integral is taken over the whole plane.

LEMma 1. The function $\widehat{\mu}(z)$ is defined for every $z$, is bounded and continuous on the plane (holomorphic outside $J$ ); moreover, there is a constant 
$K$ such that

$$
\left|\widehat{\mu}\left(z_{1}\right)-\widehat{\mu}\left(z_{2}\right)\right| \leq K\left|z_{1}-z_{2}\right| \log \left|z_{1}-z_{2}\right|^{-1}
$$

for every $z_{1}, z_{2}$.

Proof. All these properties of the Cauchy transform of an $L_{\infty}$ function with compact support are standard and can be easily verified.

Complement to Lemma 1. It can be shown that $\widehat{\mu}$ satisfies a stronger Zygmund condition: there is $M$ such that, for every $z, h$,

$$
|\widehat{\mu}(z+h)+\widehat{\mu}(z-h)-2 \widehat{\mu}(z)| \leq M|h| .
$$

In what follows, an important role is played by the following formula:

$$
\sum_{f(w)=u} \frac{1}{\left[f^{\prime}(w)\right]^{2}(z-w)}=\frac{1}{f^{\prime}(z)}\left(\frac{1}{u-c}+\frac{1}{f(z)-u}\right) .
$$

It can be easily derived e.g. by applying the Residue Theorem to the integral $(2 \pi i)^{-1} \int_{\Gamma}\left[f^{\prime}(w)(f(w)-u)(z-w)\right]^{-1} d w$, where $\Gamma$ is a contour around the infinity.

(Note that all this holds also for $f(z)=z^{d}+c$, with any integer $d \geq 2$.)

Lemma 2. $\widehat{\mu}$ satisfies the following functional equation:

$$
f^{\prime}(z) \widehat{\mu}(z)-\widehat{\mu}(f(z))=-\widehat{\mu}(c) .
$$

Proof. We use the property (2) of $\mu$, make a change of variable $u=f(w)$ in the integral which defines $\widehat{\mu}$, and then use the identity $(* *)$ :

$$
\begin{aligned}
\widehat{\mu}(z) & =\int \mu(u) \sum_{f(w)=u} \frac{1}{\left[f^{\prime}(w)\right]^{2}(z-w)} d \sigma_{u} \\
& =\frac{1}{f^{\prime}(z)} \int \mu(u)\left(\frac{1}{u-c}+\frac{1}{f(z)-u}\right) d \sigma_{u}=\frac{1}{f^{\prime}(z)}(\widehat{\mu}(f(z))-\widehat{\mu}(c)) .
\end{aligned}
$$

LEMma 3. $\widehat{\mu}(c) \neq 0$.

Proof. Assume the contrary. Then from the functional equation (4) we see that $\widehat{\mu}(z)=\widehat{\mu}\left(f^{n}(z)\right) /\left(f^{n}\right)^{\prime}(z), n>0$. If $z \notin J$, then $f^{n}(z) \rightarrow \infty$, and we get $\widehat{\mu}(z)=0$ off $J$. Since $\widehat{\mu}$ is continuous on the plane and $J$ is nowhere dense, $\widehat{\mu}$ is identically zero. But $\mu$ is a generalized derivative of $\widehat{\mu}$ with respect to $\bar{z}[\mathrm{Ah}]$, hence, it is zero as well, a contradiction.

Now we can normalize $\widehat{\mu}$ : define

$$
Q(z)=\widehat{\mu}(z) / \widehat{\mu}(c) .
$$

Thus we have

$$
f^{\prime}(z) Q(z)-Q(f(z))=-1 \quad \text { for every } z .
$$


REMARK 11. This functional equation (with a spectral parameter before $Q(f(z)))$ has already been studied in [LSY] as an equation for the Cauchy transform of eigenvectors of the adjoint operator of some Ruelle operator $T$ (see below) which was defined in [LSY] for hyperbolic maps. See also Remark 13 and references there.

LEMma 4. (a) $Q(0)=0$.

(b) If for some $z,\left(f^{n}\right)^{\prime}(z) \rightarrow \infty$ as $n \rightarrow \infty$, then

$$
Q(z)=-\left(\frac{1}{f^{\prime}(z)}+\ldots+\frac{1}{\left(f^{n}\right)^{\prime}(z)}+\ldots\right) .
$$

(c) $Q\left(c_{n}\right)$ is indeed equal to $\left(f^{n-1}\right)^{\prime}(c)\left(1+1 / f^{\prime}(c)+\ldots+1 /\left(f^{n-1}\right)^{\prime}(c)\right)$ (consistent with the definition in the Introduction).

Proof. It follows from (2) that $\mu$ is an even function. $J$ is symmetric with respect to the origin. Hence,

$$
\widehat{\mu}(0)=\int \frac{\mu(w)}{w} d \sigma_{w}=0 .
$$

This proves (a).

(b)-(c) follow from the functional equation (6) for $Q(z)$.

Parts (a)-(b) of Proposition 1 follow immediately from Lemmas 1 and 4.

REMARK 12. In fact, for any $z \in \mathbb{C}, Q(z)$ has a transparent geometrical meaning. Recall [MSS] that since $f(z)=f_{c}(z)=z^{2}+c$ has an invariant linefield, there is a neighborhood $W$ of $c$ such that, for every $\widehat{c} \in W$, the maps $f_{c}(z)$ and $f_{\widehat{c}}(z)=z^{2}+\widehat{c}$ are conjugate by a quasi-conformal homeomorphism $h_{\widehat{c}}$ of the plane. Moreover, for a.e. $z \in \mathbb{C}$, the dilatation

$$
\frac{\partial h_{\widehat{c}}(z)}{\partial \bar{z}} / \frac{\partial h_{\widehat{c}}(z)}{\partial z}
$$

is holomorphic in $\widehat{c}$. The interpretation of $Q(z)$ is now the following: for every $z$,

$$
Q(z)=\left.\frac{\partial h_{\widehat{c}}(z)}{\partial \widehat{c}}\right|_{\widehat{c}=c} .
$$

In order to prove this, we set $P(z)=\left.\left(\partial h_{\widehat{c}}(z) / \partial \widehat{c}\right)\right|_{\widehat{c}=c}$ and then observe the following. By the theory of quasi-conformal mappings [Ah], $P(z)$ is a continuous function on the plane. On the other hand, we check that if $z$ is outside the Julia set of $f_{c}, P(z)$ is holomorphic in $z$, bounded at infinity, and satisfies the same functional equation (6). All this follows easily from the expression of $h_{\widehat{c}}$ outside the Julia set as $B_{\widehat{c}}^{-1} \circ B_{c}(z)$, where $B_{\widehat{c}}$ is the Böttcher coordinate of $f_{\widehat{c}}$ outside its Julia set. Another way to see that $P$ satisfies (6) is to differentiate the identity $h_{\widehat{c}}\left(f_{c}(z)\right)=\left[h_{\widehat{c}}(z)\right]^{2}+\widehat{c}$ with respect to $\widehat{c}$. 
3. Proof of Proposition 3. For every function $g$ which is defined and holomorphic outside $J$, define

$$
(T g)(z)=\sum_{f(w)=z} \frac{g(w)}{\left(f^{\prime}(w)\right)^{2}},
$$

which is again a holomorphic function outside $J$.

LEMMA 5.

$$
\left(T \phi_{n}\right)(z)=\phi_{n}+R_{n}(z)
$$

where

$$
\begin{aligned}
R_{n}(z) & =\frac{1}{\left(f^{n}\right)^{\prime}(c)} \cdot \frac{1}{z-c_{n+1}}-\frac{S_{n+1}}{z-c_{1}} \\
& =\frac{1}{\left(f^{n-1}\right)^{\prime}(c)} \cdot \frac{c_{n}}{2(z-c)\left(z-c_{n+1}\right)}-\frac{S_{n}}{z-c} \\
& =\frac{1}{\left(f^{n-1}\right)^{\prime}(c)} \cdot T\left(\frac{1}{z-c_{n}}\right)-\frac{S_{n}}{z-c} .
\end{aligned}
$$

Proof. Let $g_{a}(z)=1 /(z-a)$, where $a \in J$ is a parameter, and $z$ is not in $J$. The identity $(* *)$ means that

$$
\left(T\left(g_{a}\right)\right)(z)=\frac{1}{f^{\prime}(a)} \cdot\left(\frac{1}{z-f(a)}-\frac{1}{z-c_{1}}\right) .
$$

Then the desired formulae are easily verified.

Choose a bounded neighborhood $V$ of $J$ such that $U=f^{-1}(V)$ is contained in $V$.

Now we use an idea which is somewhat similar to [Ts]. We can write

$$
\begin{aligned}
\int_{V \backslash J}\left|\phi_{n}(z)\right| d \sigma_{z} & =\int_{V \backslash J}\left|\left(T \phi_{n}\right)(z)-R_{n}(z)\right| d \sigma_{z} \\
& \leq \int_{V \backslash J f(w)=z} \frac{\left|\phi_{n}(w)\right|}{\left|f^{\prime}(w)\right|^{2}} d \sigma_{z}+\int_{V \backslash J}\left|R_{n}(z)\right| d \sigma_{z} \\
& =\int_{U \backslash J}\left|\phi_{n}(z)\right| d \sigma_{z}+\int_{V \backslash J}\left|R_{n}(z)\right| d \sigma_{z} .
\end{aligned}
$$

Therefore,

$$
\begin{aligned}
\int_{V \backslash U}\left|\phi_{n}(z)\right| d \sigma_{z} & \leq \int_{V \backslash J}\left|R_{n}(z)\right| d \sigma_{z} \\
& \leq \frac{1}{\left|\left(f^{n-1}\right)^{\prime}(c)\right|} \int_{V \backslash J}\left|T \frac{1}{z-c_{n}}\right| d \sigma_{z}+\left|S_{n}\right| \int_{V \backslash J} \frac{d \sigma_{z}}{\left|z-c_{1}\right|} \\
& \leq \frac{1}{\left|\left(f^{n-1}\right)^{\prime}(c)\right|} \int_{U \backslash J} \frac{d \sigma_{z}}{\left|z-c_{n}\right|}+\left|S_{n}\right| \int_{V \backslash J} \frac{d \sigma_{z}}{\left|z-c_{1}\right|} .
\end{aligned}
$$


Given the domain $V$, find a constant $M_{4}$ such that, for every $a \in V$,

$$
\int_{V} \frac{d \sigma_{w}}{|w-a|} \leq M_{4}
$$

Then

$$
\int_{V \backslash U}\left|\phi_{n}(z)\right| d \sigma_{z} \leq M_{4}\left(\frac{1}{\left|\left(f^{n-1}\right)^{\prime}\left(c_{1}\right)\right|}+\left|S_{n}\right|\right) .
$$

Assume (from now on) that $f$ has an invariant linefield on $J$. Then, by Proposition 1(a), $\left|S_{n}\right| \leq M_{1} /\left|\left(f^{n-1}\right)^{\prime}\left(c_{1}\right)\right|$. Therefore,

$$
\int_{V \backslash U}\left|\phi_{n}(z)\right| d \sigma_{z} \leq \frac{M_{2}}{\left|\left(f^{n-1}\right)^{\prime}\left(c_{1}\right)\right|},
$$

where $M_{2}=M_{4}\left(1+M_{1}\right)$, which proves part (a) of Proposition 3 .

To prove part (b), note that any compact set $K$ disjoint from $J$ can be covered by finitely many domains of the form $V \backslash f^{-1}(V)$, where $V$ is as in part (a). Therefore, part (b) will be an immediate consequence of (a) and the following well known simple fact.

Lemma 6. Let $\Omega$ be a domain of the plane, and $K \subset \Omega$ a compact set. Then there exists a constant $M$ such that for every function $g$ holomorphic in $\Omega$, and every $z \in K$,

$$
|g(z)| \leq M \int_{\Omega}|g(w)| d \sigma_{w} .
$$

Proof. Let $\delta$ be the distance between $K$ and $\partial \Omega$. For $z \in K$, we have

$$
g(z)=\frac{1}{\pi \delta^{2}} \int_{D(z, \delta)} g(w) d \sigma_{w}
$$

so

$$
|g(z)| \leq \frac{1}{\pi \delta^{2}} \int_{D(z, \delta)}|g(w)| d \sigma_{w} \leq \frac{1}{\pi \delta^{2}} \int_{\Omega}|g(w)| d \sigma_{w},
$$

and we can take $M=1 /\left(\pi \delta^{2}\right)$.

4. Proof of Theorem 1 and Corollary 1(b). So, we assume that $f$ has an invariant linefield on $J$, and that

$$
\left(f^{n_{i}-1}\right)^{\prime}(c) \Delta_{n_{i}} \rightarrow \infty
$$

Recall that

$$
\Delta_{n}=1+\frac{1}{\left|f^{\prime}(c)\right|}+\ldots+\frac{1}{\left|\left(f^{n-1}\right)^{\prime}(c)\right|}
$$




$$
\nu_{n}=\frac{1}{\Delta_{n}} \sum_{k=1}^{n} \frac{1}{\left(f^{k-1}\right)^{\prime}(c)} \delta_{c_{k}} .
$$

Introduce functions $\psi_{n}$ defined and holomorphic outside $J$ as follows:

$$
\psi_{n}(z)=\frac{1}{\Delta_{n}} \phi_{n}(z)
$$

Then one can write

$$
\psi_{n}(z)=\int_{J} \frac{d \nu_{n}(w)}{z-w}
$$

On the other hand, it follows from Proposition 3(b), and the assumption, that the sequence $\psi_{n_{i}}$ tends to zero uniformly on compact sets outside $J$. Since the total variation of each measure $\nu_{n}$ is equal to 1 , every subsequence of $\nu_{n}$ contains a converging subsubsequence. So, we have to prove that if a subsequence of $\nu_{n_{i}}$ converges to a measure $\nu$, then $\nu=0$. From the above we deduce that the function

$$
\int_{J} \frac{d \nu(w)}{z-w}
$$

is equal to zero for every $z$ outside $J$.

The following statement was pointed out to me by P. Makienko (cf. [Ga, Ch. 2, Th. 8.1]):

LEMMA 7. Let $m$ be a complex measure on the plane with a compact support $K$ such that any continuous function on $K$ can be uniformly approximated by rational functions with poles outside $K$. If

$$
\int \frac{d m(w)}{z-w}=0
$$

outside $K$, then $m=0$.

Proof. If $r$ is any rational function as above, then choosing a contour $\gamma$ enclosing $K$ close enough to $K$ and applying Fubini's theorem shows that

$$
\int r(z) d m(z)=\int d m(z) \frac{1}{2 \pi i} \int_{\gamma} \frac{r(w)}{z-w} d w=\frac{1}{2 \pi i} \int_{\gamma} r(w) d w \int \frac{d m(z)}{z-w} d z=0 .
$$

If now $g$ is continuous on $K$, for any $\varepsilon>0$ find $r$ as above such that $|g(z)-r(z)|<\varepsilon /|m|$ on $K$, where $|m|$ is the total variation of $m$. Then $\left|\int g(z) d m(z)\right| \leq\left|\int r(z) d m(z)\right|+\varepsilon=\varepsilon$. Thus $\int g d m=0$ for any continuous function on $K$, and the statement follows.

Since $f$ has an invariant linefield, the Julia set $J$ does not separate the plane. Therefore, by Mergelyan's theorem [Ga] the compact set $K=J$ satisfies the condition of Lemma 7. As a consequence, we conclude that $\nu=0$. 
Let us prove Corollary 1(b) by contradiction. Assume that the sequence $S_{n}$ converges to zero. Introduce the following (complex) measure:

$$
\nu^{\prime}=\sum_{k=1}^{\infty} \frac{1}{\left(f^{k-1}\right)^{\prime}(c)} \delta_{c_{k}} .
$$

The definition is correct, because the series $\sum_{k>0} 1 /\left|\left(f^{k-1}\right)^{\prime}(c)\right|$ converges. Now define

$$
\phi(z)=\int_{J} \frac{d \nu^{\prime}(w)}{z-w} .
$$

Then:

(1) $\phi$ is a holomorphic function in each component of $\mathbb{C} \backslash J$, and $\phi$ is locally integrable on the plane.

Since $S_{n} \rightarrow 0$, the formula of Lemma 5 shows that

(2) $T \phi=\phi$ off $J$.

Claim. For every quadratic polynomial $f$, the conditions (1)-(2) above imply $\phi=0$ off $J$.

Proof. Let $\Omega$ be a component of $\mathbb{C} \backslash J$. We prove that $\phi=0$ on $\Omega$. If $\Omega$ is the unbounded component, then we choose an open set $V$ in $\Omega$ such that $U=f^{-1}(V)$ is a subset of $V$ and $V \backslash U$ is a non-empty open set. We know that $T \phi=\phi$ implies $\int_{V}|\phi| d \sigma \leq \int_{U}|\phi| d \sigma$, i.e. $\int_{V \backslash U}|\phi| d \sigma=0$, hence, by Lemma $6, \phi=0$ on $\Omega$. If $\Omega$ is a bounded component of $\mathbb{C} \backslash J$, set $V_{\Omega}=\bigcup_{n \geq 0} f^{-n}(\Omega)$. If $\Omega$ is not periodic (just eventually periodic), then $V_{\Omega} \backslash f^{-1}\left(V_{\Omega}\right)=\Omega$. Then again $\int_{\Omega}|\phi| d \sigma=0$, and $\phi=0$ on $\Omega$. Finally, let $\Omega$ be a periodic component of period $p>0: f^{p}(\Omega)=\Omega$. Then $\Omega$ is a Siegel disc, and $f^{-1}(\Omega)$ consists of two different components, one of which is not periodic. By the above, $\phi=0$ on this component. Since $T \phi=\phi$, this implies that $\phi\left(f^{p}(w)\right)\left[\left(f^{p}\right)^{\prime}(w)\right]^{2}=\phi(w)$ for every $w \in \Omega$. Conjugating $f^{p}$ with an irrational rotation, we see that again $\phi=0$ on $\Omega$. The claim is proved.

Thus $\phi=0$ outside $J$. By [Ga, Ch. 8, Corr. 8.4], the compact set $J$ satisfies the condition of Lemma 7 . By Lemma $7, \nu^{\prime}=0$, a contradiction.

REMARK 13. Corollary 1(b) can be generalized at least in two directions. One of them is the generalization to rational functions which satisfy the summability condition at the critical points in the Julia set. Let us describe another one. Namely, let us show that under the condition of Corollary 1, for any complex number $\lambda$ such that $|\lambda| \leq 1$, we have

$$
D(\lambda) \neq 0, \quad \text { where } \quad D(\lambda)=1+\sum_{n=1}^{\infty} \frac{\lambda^{n}}{\left(f^{n}\right)^{\prime}(c)} .
$$


The proof is essentially the same as above: fix $\lambda$ with $|\lambda| \leq 1$, and let

$$
\phi_{\lambda}(z)=\sum_{k=1}^{\infty} \frac{\lambda^{k-1}}{\left(f^{k-1}\right)^{\prime}(c)\left(z-c_{k}\right)} .
$$

If $D(\lambda)=0$, then $\lambda \cdot T \phi_{\lambda}=\phi_{\lambda}$, and hence $\phi_{\lambda}=0$ off $J$, again a contradiction with Lemma 7 (see also proof in the next section). This proof even shows that $D(\lambda) \neq 0$ inside the circle of convergence without the summability condition, provided $|\lambda| \leq 1$. Note that $D$ is the Fredholm determinant of the (Ruelle) operator $T$ in an appropriate function space (cf. [LSY], [LSY1], [Le1], [ELS]).

5. Proof of Theorem 2. We prove first part (b) and then derive (a) from (b). So, assume that the series

$$
1+\sum_{n \geq 1}\left(f^{n}\right)^{\prime}\left(c_{-n}\right)
$$

converges absolutely, and denote by $S^{*}$ its sum. Introduce the function

$$
\phi_{-}(z)=\frac{1}{z}+\sum_{n=1}^{\infty} \frac{\left(f^{n}\right)^{\prime}\left(c_{-n}\right)}{z-c_{-n}},
$$

defined and holomorphic outside $J$. Now we continue as in the proofs above. First, check that

$$
\left(T \phi_{-}\right)(z)=\phi_{-}(z)-\frac{S^{*}}{z-c_{1}} .
$$

Assume now that $S^{*}=0$. Then $T \phi_{-}=\phi_{-}$. Applying the Claim from the previous section, we find that $\phi_{-}=0$ outside $J$. On the other hand,

$$
\phi_{-}(z)=\int \frac{d \nu_{-}(w)}{z-w}
$$

with the measure

$$
\nu_{-}=\delta_{c_{0}}+\sum_{n \geq 1}\left(f^{n}\right)^{\prime}\left(c_{-n}\right) \delta_{c_{-n}}
$$

a contradiction with Lemma 7.

Thus we have proved that $S^{*} \neq 0$.

To finish the proof of part (b), we assume that $f$ does have an invariant linefield, and show that this implies $S^{*}=0$. Indeed, then the functional equation (6) for the function $Q$ gives us

$$
1+\sum_{k=1}^{n-1}\left(f^{k}\right)^{\prime}\left(c_{-k}\right)=-\left(f^{n}\right)^{\prime}\left(c_{-n}\right) Q\left(c_{-n}\right) .
$$


Since $\sum_{n \geq 1}\left|\left(f^{n}\right)^{\prime}\left(c_{-n}\right)\right|<\infty$, it follows that $\left(f^{n}\right)^{\prime}\left(c_{-n}\right) \rightarrow 0$. Clearly, by Proposition 1, $Q$ is bounded. Hence, $\left(f^{n}\right)^{\prime}\left(c_{-n}\right) Q\left(c_{-n}\right) \rightarrow 0$, and we conclude that $S^{*}=0$, a contradiction.

To derive (a) from (b), assume that there is $M$ such that, for every $i$,

$$
\left|\left(f^{n_{i}-1}\right)^{\prime}(c)\right| \Delta_{n_{i}}=1+\sum_{k=1}^{n_{i}-1}\left|\left(f^{k}\right)^{\prime}\left(c_{n_{i}-k}\right)\right|<M .
$$

Using the standard diagonal procedure and passing to a subsequence, one can assume that, for every $k$, the sequence $c_{n_{i}-k}$ converges to some point $c_{-k}$ so that $f\left(c_{-k}\right)=c_{-(k-1)}, k \geq 1$, and $c_{0}=0$. It is easy to see that

$$
1+\sum_{k=1}^{\infty}\left|\left(f^{k}\right)^{\prime}\left(c_{-k}\right)\right| \leq M
$$

because for every fixed $N$,

$$
1+\sum_{k=1}^{N}\left|\left(f^{k}\right)^{\prime}\left(c_{-k}\right)\right|=\lim _{i \rightarrow \infty}\left(1+\sum_{k=1}^{N}\left|\left(f^{k}\right)^{\prime}\left(c_{n_{i}-k}\right)\right|\right) \leq M .
$$

It remains to apply part (b).

6. Proof of Proposition 4. Let $\Omega$ be a Y-domain and $a$ be a point of $\partial \Omega \cap J$. Since the point $a$ is (pre)periodic, there exists a sequence of puzzle pieces of $f$ which shrink to $a$. Therefore and because of Proposition 3(b), it is enough to prove the statement assuming that $\Omega$ is one of the puzzle pieces disjoint from $\omega$. Moreover, one can assume that there is a neighborhood $\Omega^{\prime}$ of $\Omega$ also disjoint from $\omega$. Hence, every $\phi_{n}$ is holomorphic in $\Omega^{\prime}(\omega$ contains all $c_{j}$ ). In view of Lemma 6 , it is enough to find a constant $M$ such that, for all $n$,

$$
\int_{\Omega}\left|\phi_{n}(z)\right| d \sigma_{z} \leq \frac{M}{\left|\left(f^{n-1}\right)^{\prime}(c)\right|} .
$$

(Strictly speaking, the required estimate will then hold not for $\Omega$ itself but for the puzzle pieces inside $\Omega$.)

We estimate the latter integral, say $I$, in two steps.

Step 1. First, we express $\Omega$ as a union of pairwise disjoint sets $E_{k} \backslash E_{k+1}$, $k=0,1, \ldots$ (such that $E_{k+1} \subset E_{k}$ ), and the rest $\bigcap_{k \geq 0} E_{k}$, which is a set of zero area. Hence,

$$
\int_{\Omega}\left|\phi_{n}(z)\right| d \sigma_{z}=\sum_{k=0}^{\infty} I_{k}
$$

where

$$
I_{k}=\int_{E_{k} \backslash E_{k+1}}\left|\phi_{n}(z)\right| d \sigma_{z}
$$


Second, for every $k$ we find an open set $V_{k}$ such that

$$
I_{k} \leq \frac{M_{8}}{\left|\left(f^{n-1}\right)^{\prime}\left(c_{1}\right)\right|} C_{k}
$$

where $M_{8}$ is an absolute constant, and

$$
C_{k}=\sup _{j>0} \int_{V_{k}} \frac{d \sigma_{z}}{\left|z-c_{j}\right|} .
$$

It is easy to see that the supremum is finite.

Step 2. Then we show that $C_{k}$ tends to zero at least exponentially fast as $k \rightarrow \infty$.

Steps 1-2 will prove the statement.

To construct the sets $E_{k}$ and $V_{k}$, we need some general (and well known) properties of first return maps.

Let $E$ be any open set. Denote by $D_{E}$ the set of points $x$ such that there is $n>0$ so that $f^{n}(x) \in E$. Then define the first return map $R_{E}: D_{E} \rightarrow E$ to $E$ as

$$
R_{E}(x)=f^{p}(x),
$$

where $p>0$ is the minimal $n$ as above. From now on $E$ will be a collection of disjoint puzzle pieces. Then every component of $D_{E}$ is either disjoint from or contained in $E$. Define

$$
D_{E}^{0}=D_{E} \cap E, \quad D_{E}^{\infty}=D_{E} \backslash D_{E}^{0},
$$

and let $f_{E}$ be the restriction of $R_{E}$ to $D_{E}^{0}$. Note that $D_{E}^{0}=f_{E}^{-1}(E)$. Let also $V(E)=E \cup D_{E}=E \cup D_{E}^{\infty}$. The following two lemmas are quite standard and easy to verify:

LEMMA 8.

1. $f^{-1}(V(E)) \subset V(E)$.

2. $V(E) \backslash f^{-1}(V(E))=E \backslash D_{E}^{0}$.

LEMMA 9.

1. $D_{D_{E}^{0}}=R_{E}^{-1}\left(D_{E}^{0}\right)$.

2. $R_{D_{E}^{0}}=\left.R_{E}\right|_{R_{E}^{-1}\left(D_{E}^{0}\right)}$.

Step 1. Define now $E_{0}=\Omega, E_{1}=D_{E_{0}}^{0}, \ldots, E_{k}=D_{E_{k-1}}^{0}, \ldots$ Then $E_{k} \subset E_{k-1}$. From Lemma $9, D_{E_{k}}=R_{E}^{-1}\left(E_{k}\right)$ and $E_{k}=f_{E}^{-k}(\Omega)$. Set further $V_{k}=V\left(E_{k}\right)=E_{k} \cup D_{E_{k}}$. Then $V_{k}=E_{k} \cup R_{E}^{-1}\left(E_{k}\right)$. From Lemma 8,

$$
f^{-1}\left(V_{k}\right) \subset V_{k} \quad \text { and } \quad V_{k} \backslash f^{-1}\left(V_{k}\right)=E_{k} \backslash E_{k+1} .
$$


This allows us to repeat an estimate of the proof of Lemma 5 and write

$$
\begin{aligned}
I_{k} & =\int_{E_{k} \backslash E_{k+1}}\left|\phi_{n}(z)\right| d \sigma_{z} \\
& \leq \frac{1}{\left|\left(f^{n-1}\right)^{\prime}\left(c_{1}\right)\right|} \int_{f^{-1}\left(V_{k}\right)} \frac{d \sigma_{z}}{\left|z-c_{n}\right|}+\frac{M_{1}}{\left|\left(f^{n-1}\right)^{\prime}\left(c_{1}\right)\right|} \int_{V_{k}} \frac{1}{\left|z-c_{1}\right|} d \sigma_{z} \\
& \leq \frac{M_{8}}{\left|\left(f^{n-1}\right)^{\prime}\left(c_{1}\right)\right|} C_{k},
\end{aligned}
$$

where $M_{8}=M_{1}+1$ is an absolute constant (depending on $f$ ), and

$$
C_{k}=\sup _{j>0} \int_{V_{k}} \frac{d \sigma_{z}}{\left|z-c_{j}\right|} .
$$

This finishes Step 1.

Step 2. Let us prove the statements about the measure of the set $\bigcap_{k>0} E_{k}$ and the estimates of $C_{k}$. Let $U$ be any component of $D_{E_{k}}$. Then an iterate $f^{q}$ of $f$ maps $U$ univalently onto $\Omega$, and, moreover, a neighborhood $U^{\prime}$ of $U$ onto the neighborhood $\Omega^{\prime}$ of $\Omega$. Therefore, the distortion of $f^{q}$ on $U$ is bounded uniformly over all $U$ and all $k$. If now $W$ is the union of all components of $D_{E_{k+1}}$ which are contained in the component $U$ of $D_{E_{k}}$, then $U \backslash W$ is mapped by $f^{q}$ onto $\Omega \backslash E_{1}$ with uniformly bounded distortion. Hence, if $|A|$ denotes the area of a set $A$, there is a universal $\lambda_{0}$ between 0 and 1 such that $|U \backslash W|>\lambda_{0}|U|$. In particular, it follows that $\left|E_{k}\right|<\left(1-\lambda_{0}\right)^{k}|\Omega|, k>0$. This shows that $\bigcap_{k \geq 0} E_{k}$ has zero area.

Let us pass to the estimate of $C_{k}$. The set $V_{k}$ consists of infinitely many components $U_{i}^{k}, i \in \Lambda_{k}$. Denote by $L_{i, k}$ the Euclidean distance between $U_{i}^{k}$ and the set $\omega$ (which contains all $c_{j}$ ), and by $d_{i, k}$ the diameter of $U_{i, k}$. Bounded distortion considerations show that there is an absolute positive constant $\lambda_{1}$ such that $L_{i, k}>\lambda_{1} d_{i, k}$ for all $k$ and $i$. Let $d(A, b)$ denote the distance between a set $A$ and a point $b$. Then, for each $c_{j}$ and all $z \in U_{i}^{k}$,

$$
\left|z-c_{j}\right|<d\left(U_{i}^{k}, c_{j}\right)+d_{i, k}<d\left(U_{i}^{k}, c_{j}\right)+\frac{L_{i, k}}{\lambda_{1}} \leq\left(1+\frac{1}{\lambda_{1}}\right) d\left(U_{i}^{k}, c_{j}\right),
$$

and, hence,

$$
\lambda_{2} \frac{\left|U_{i}^{k}\right|}{d\left(U_{i}^{k}, c_{j}\right)} \leq \int_{U_{i}^{k}} \frac{d \sigma_{z}}{\left|z-c_{j}\right|} \leq \frac{\left|U_{i}^{k}\right|}{d\left(U_{i}^{k}, c_{j}\right)},
$$

where $\lambda_{2}=1 /\left(1+1 / \lambda_{1}\right)$. Therefore,

$$
\lambda_{2} \Sigma(k, j) \leq \int_{V_{k}} \frac{d \sigma_{z}}{\left|z-c_{j}\right|} \leq \Sigma(k, j),
$$


where

$$
\Sigma(k, j)=\sum_{i \in \Lambda_{k}} \frac{\left|U_{i}^{k}\right|}{d\left(U_{i}^{k}, c_{j}\right)} .
$$

Since

$$
C_{k}=\sup _{j>0} \int_{V_{k}} \frac{d \sigma_{z}}{\left|z-c_{j}\right|}
$$

is finite, we see that

$$
\Sigma(k)=\sup _{j>0} \Sigma(k, j)
$$

is finite as well, and

$$
\lambda_{2} \Sigma(k) \leq C_{k} \leq \Sigma(k)
$$

for every $k \geq 0$. Let us estimate $\Sigma(k+1) / \Sigma(k)$. Let $U_{r}^{k+1}$ be all components of $V_{k+1}$ which are contained in $U_{i}^{k}$. Then we know that $\sum_{r}\left|U_{r}^{k+1}\right| \leq$ $\left(1-\lambda_{0}\right)\left|U_{i}^{k}\right|$. On the other hand, for every $c_{j}$ and every $r, d\left(U_{r}^{k+1}, c_{j}\right) \geq$ $d\left(U_{i}^{k}, c_{j}\right)$. Hence, $\Sigma(k+1, j) \leq\left(1-\lambda_{0}\right) \Sigma(k, j)$, and finally

$$
\Sigma(k+1) \leq\left(1-\lambda_{0}\right) \Sigma(k) \leq\left(1-\lambda_{0}\right)^{k} \Sigma(0),
$$

that is,

$$
\sum_{k \geq 0} C_{k} \leq \sum_{k \geq 0} \Sigma(k) \leq \frac{\Sigma(0)}{\lambda_{0}} .
$$

This completes Step 2 and the proof of Proposition 4.

7. Proof of Corollary 4, Proposition $\mathbf{3}^{\prime}$, and Theorem $\mathbf{1}^{\prime}$. Corollary 4 is an immediate consequence of Proposition 4 because any compact set $K$ outside $Y(\omega)$ is covered by finitely many puzzle pieces which are disjoint from $Y(\omega)$.

Let us prove Proposition $3^{\prime}$. Given a compact set $K$ outside $J^{\prime}$, find a puzzle piece $\Omega$ such that the closure of $\Omega$ is disjoint from $K, J^{\prime}$ is contained in the closure of $\Omega$, and $\Omega$ contains only those iterates of the critical point which are inside $J^{\prime}$. Let $\gamma=\partial \Omega$. Note that $\gamma \cap Y(\omega)=\emptyset$. Then, by Corollary 4, there is $M$ such that for all $n$ and $z \in \gamma$,

$$
\left|\phi_{n}(z)\right| \leq \frac{M}{\left|\left(f^{n-1}\right)^{\prime}(c)\right|} .
$$

Therefore, for any $a \in K$ we can write

$$
\left|\phi_{n, J^{\prime}}(a)\right|=\left|\frac{1}{2 \pi i} \int_{\gamma} \frac{\phi_{n}(z)}{a-z} d z\right| \leq \frac{M_{6}}{\left|\left(f^{n-1}\right)^{\prime}(c)\right|},
$$

with $M_{6}=(2 \pi)^{-1} L M / d(K, \gamma)$, where $L$ is the length of $\gamma$ and $d(K, \gamma)$ is the distance between the disjoint compact sets $K$ and $\gamma$. 
In the same way, we estimate

$$
S_{n, J^{\prime}}=\frac{1}{2 \pi i} \int_{\gamma} \phi_{n}(z) d z .
$$

This proves (a)-(b).

Theorem $1^{\prime}$ is proved similarly to the proof of Theorem 1 using Proposition $3^{\prime}(\mathrm{a})$.

\section{References}

[Ah] L. Ahlfors, Lectures on Quasiconformal Mappings, Van Nostrand, 1966.

[BS] H. Bruin and S. van Strien, Expansion of derivatives in one-dimensional dynamics, preprint, Sept. 2000.

[CG] L. Carleson and T. Gamelin, Complex Dynamics, Springer, 1993.

[DH] A. Douady et J. H. Hubbard, Étude dynamique des polynômes complexes, Partie I, Publ. Math. Orsay 84-2, 1984.

[ELS] A. Eremenko, G. Levin and M. Sodin, On the distribution of zeros of a Ruelle zeta-function, Comm. Math. Phys. 159 (1994), 433-441.

[Ga] T. Gamelin, Uniform Algebras, Prentice-Hall, 1969.

$[\mathrm{H}] \quad$ J. H. Hubbard, Local connectivity of Julia sets and bifurcation loci: three theorems of J.-C. Yoccoz, in: Topological Methods in Modern Mathematics (Stony Brook, NY, 1991), Publish or Perish, 1993, 467-511.

[Le] G. Levin, On backward stability of holomorphic dynamical systems, Fund. Math. 158 (1998), 97-107.

[Le1] - On Mayer's conjecture and zeros of entire functions, Ergodic Theory Dynam. Systems 14 (1994), 565-574.

[LSY] G. Levin, M. Sodin and P. Yuditskiı̌, Ruelle operators with rational weights for Julia sets, J. Anal. Math. 63 (1994), 303-331.

[LSY1] - - - 一, A Ruelle operator for a real Julia set, Comm. Math. Phys. 141 (1991), $119-132$.

[Mak] P. Makienko, Remarks on Ruelle operator and invariant line fields problem, preprint, July 2000.

[Mc] C. McMullen, Complex Dynamics and Renormalization, Ann. of Math. Stud. 135, Princeton Univ. Press, 1994.

[MSS] R. Mañé, P. Sad and D. Sullivan, On the dynamics of rational maps, Ann. Sci. École Norm. Sup. 16 (1983), 193-217.

[R-L] J. Rivera-Letelier, Rational maps with decay of geometry: rigidity, Thurston's algorithm and local connectivity, Stony Brook preprint 2000/9.

[Ts] M. Tsujii, A simple proof for monotonicity of entropy in the quadratic family, Ergodic Theory Dynam. Systems 20 (2000), 925-933.

Institute of Mathematics

The Hebrew University

Givat Ram 91904, Jerusalem, Israel

E-mail: levin@math.huji.ac.il 